\title{
ON A RIEMANN SUM CONSTRUCTION OF RUDIN
}

\author{
C. J. MOZZOCHI
}

There is a slight error on p. 323 of [1] where Rudin states "But $n_{j}$ does not divide $k_{j} p_{j} \ldots$ and therefore the terms of the arithmetic progression

$$
k_{j} p_{j}\left(x+\left(r / n_{j}\right)\right) \quad\left(r=1,2, \cdots, n_{j}\right)
$$

are not all congruent $(\bmod 1)$. Since $\delta>\frac{1}{2}$ it follows that at least half of them lie in $G$." The idea underlying the last statement is the following: If there are $t$ equally spaced points on a circle of circumference 1 , then an interval of length $\delta>\frac{1}{2}$ should contain at least half of them. This is in fact true for even $t$ and for sufficiently large $t, t>t_{0}(\delta)$. However for $t=3$ one must have $\delta>\frac{2}{3}$. In fact one can easily verify the following:

LEMMA. If $P$ is a regular polygon inscribed in a circle of circumference 1 , then any arc of this circle of length $\delta>\frac{2}{3}$ contains at least half the vertices of $P$.

The results of [1] remain unaffected, if the condition " $\delta>\frac{1}{2}$ " is changed into " $\delta>\frac{2}{3}$ ".

\section{REFERENCE}

1. W. Rudin, An arithmetic property of Riemann sums, Proc. Amer. Math. Soc. 15 (1964), 321-324.

Trinity College

Received by the editors January 6, 1969. 